\title{
Self-administration of Pentobarbital by means of schedule-induced polydipsia
}

\author{
RICHARD A. MEISCH, ${ }^{2}$ University of \\ Minnesota, Minneapolis, Minn. 55455
}

Rats self-administered large quantities of sodium pentobarbital when a solution of the drug was substituted for water during schedule-induced polydipsia. Changes in the food baseline indicated that the drug was affecting the animals' behavior. The pattern and time course of responding for the drug were measured by making presentation of the drug contingent upon a lever press.

Rats have been induced to orally self-administer sodium pentobarbital when their source of liquid is restricted to such a solution (Crossland \& Leonard, 1963; Essig, 1966). Using this method it is not possible to separate the variable of liquid deprivation from other factors that may affect drug consumption. Lester (1961) and Senter \& Sinclair (1967) have induced rats to self-administer ethanol by substituting it for drinking water during schedule-induced polydipsia. Schedule-induced polydipsia is a term for a behavioral phenomenon originally described by Falk (1961). He found that rats will drink large quantities of water if placed on an intermittent schedule of food reinforcement. The purpose of the present study was to determine if rats would self-administer sodium pentobarbital during schedule-induced polydipsia. Unlike previous drug self-administration polydipsia studies (Lester, 1961; Senter \& Sinclair, $1967)$, changes in the food-reinforced baseline behavior were concurrently examined to determine the effects of the self-administered drug on the animals' behavior. Also, time course and pattern of responding for the drug were measured by making liquid presentation contingent upon a lever press.

\section{SUBJECTS}

The Ss were two naive male Holtzman albino rats, Ss 270 and 280 , maintained at 428 and $480 \mathrm{~g}$, respectively, which was $80 \%$ of their free-feeding weight. They were approximately 200 days old and were individually housed in a temperature controlled, continually illuminated room. Water was always available in the animals' home cages.

\section{APPARATUS}

The apparatus was a standard operant conditioning chamber, housed in a ventilated, sound-shielding enclosure. White masking noise was constantly present. Two levers, a food magazine, and a dipper for presentation of liquid were located along one wall of the conditioning chamber. The levers were separated by the reinforcement delivery mechanisms, with the food magazine directly above the dipper. Each operation of the food magazine produced a single 45-mg Noyes food pellet, and the operation of the dipper made available $0.25 \mathrm{ml}$ of liquid for $4 \mathrm{sec}$. Programming and data recording were automatic, by electromechanical equipment located in an adjacent room.

\section{PROCEDURE}

The animals were placed in the operant conditioning chamber for $6 \mathrm{~h}$ each day. On the first training day the rats were shaped to press the left lever for a food pellet. After approximately 50 consecutive food reinforcements the animals were shifted to a VI $1-$ min schedule. Responding for water by pressing the right lever was spontaneously acquired by both rats during the first session. For the duration of the experiment the animals received food pellets on a VI 1 -min schedule and liquid following each response.

The animals were trained for 2 weeks before the start of the drug sessions. On drug days the animals responded on the liquid lever for water during the first hour, and then a drug solution was substituted for water, and the animals responded for the drug solution for the remaining $5 \mathrm{~h}$. On control days only water was available as the drinking fluid for the entire 6-h session. However, at the end of the first hour the water reservoir was exchanged for another water reservoir. A control day preceded and succeeded each drug day. At least 2 days intervened between drug days. Responding on the food lever on both drug and control days always produced food on a VI 1-min schedule.

At hourly intervals, food and liquid responses and food reinforcements were recorded. Volume of liquid consumed was measured at the end of the first and sixth hours by calculating the difference between the volume originally in the reservoir and the volume remaining. Drug solutions were 0.4 , 0.8 , and $1.6 \mathrm{mg} / \mathrm{ml}$ of sodium pentobarbital (salt). The order of presentation was 0.4 , 1.6 , and $0.8 \mathrm{mg} / \mathrm{ml}$ for Rat 270 and $1.6,0.4$, and $0.8 \mathrm{mg} / \mathrm{ml}$ for Rat 280 .

\section{RESULTS}

The rats self-administered sodium pentobarbital in quantities sufficient to affect the food baseline. The number of liquid responses and volume of liquid consumed decreased as the drug concentration was increased.

Figure 1 summarizes the session totals of three dependent variables for Rats 270 and 280. The zero per cent column represents the average value of six control sessions. These control sessions preceded and succeeded each drug day.

For Rat 270 the lowest concentration produced a decreased rate of responding for food. Increasing the concentration resulted in a rate increase. For Rat 280, the lowest and highest concentrations resulted in an increased rate of food responding with no change occurring at the intermediate concentration.

In general, the number of liquid responses decreased as the drug concentration was increased. A similar relation was found to hold between volume of liquid consumed and drug concentration.

The data were analyzed using Student's $t$ test. Each experimental value was compared with the mean of the six control values. Significant differences are indicated in the figure by asterisks. The number of food reinforcements obtained during each session was also recorded. However, the only significant difference occurred at $0.4 \mathrm{mg} / \mathrm{cc}$ for Rat 270 when there was a decrease $(\mathrm{t}=7.4, \mathrm{df}=5, \mathrm{p}<.001)$.

The time course data for Rat 270 are illustrated in Fig. 2. The $0.0 \mathrm{mg} / \mathrm{cc}$ concentration data are the average of the six control days. The results for the other concentrations are the values obtained from a single session. While a significant depression occurred during Hours 3 and $4(t=4.5$ and $3.9, p<.01$ and $p<.05$ ) this was not reflected by a significant change in the food baseline total session value. As the concentration increased, responding for liquid decreased earlier in the session.

The time course of Rat 280's responses for liquid were similar to Rat 270's. However, its pattern of responding for food showed only increases and no depressions. DISCUSSION

These results demonstrate that scheduleinduced polydipsia can be used to induce rats to drink a drug other than ethanol. This is particularly significant since ethanol has caloric value for the food-deprived animal. Further, changes in the food baseline can be examined to determine whether the selfadministered drug is affecting the animal's behavior. Finally, the time course of drug self-administration can be recorded by making it contingent upon a lever press. This contingency also permits the possibility of studying schedules of drug selfadministration. Previous studies (Lester, 1961; Senter \& Sinclair, 1967) have not reported responding either for food or liquid under both drug and control conditions. 


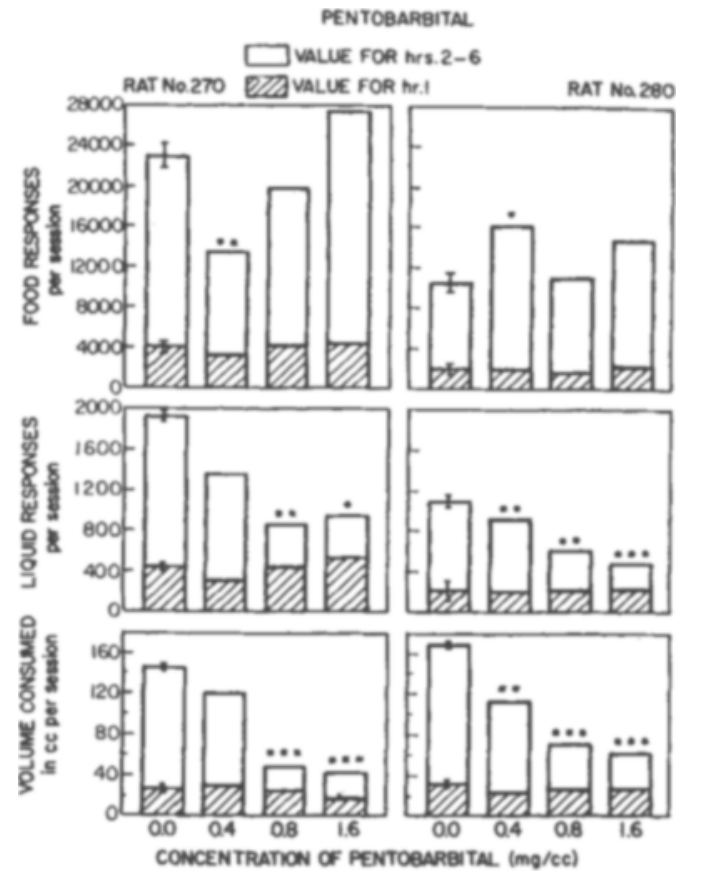

Fig. I. Session totals of food and liquid responses and volume consumed as a function of drug concentration. The standard error of the mean is indicated by the brackets. The striped section at the bottom of each column represents the value for the first hour when water was the drinking liquid. The heights of the bars for the three drug concentrations represent the value for a single 6-h session. ${ }^{*} p<.05,{ }^{* *} p<.01,{ }^{* * *} p<.001$.

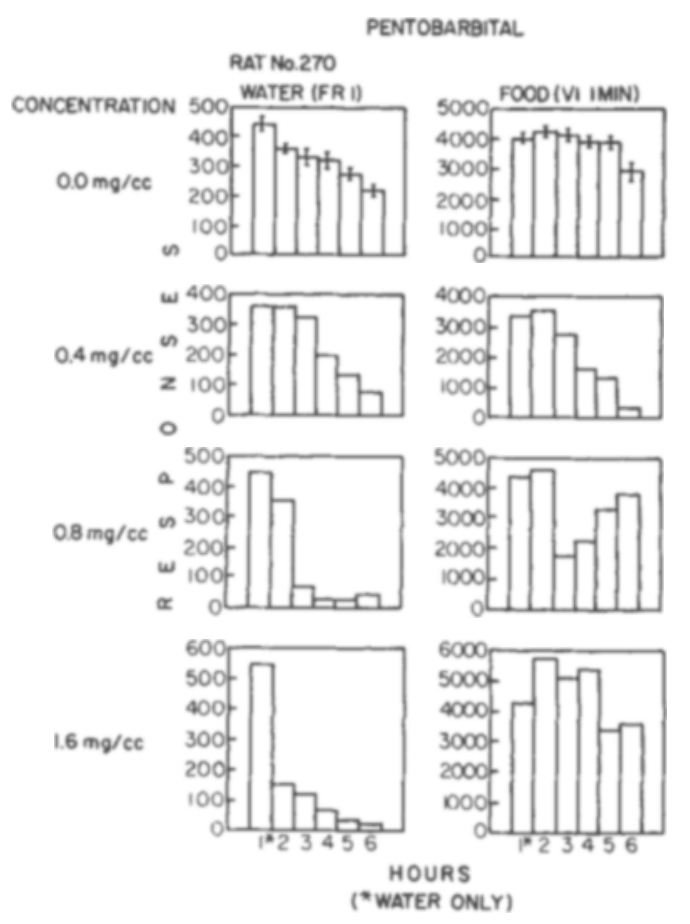

Fig. 2. Time course of responding for liquid and food by Rat 270 as a function of drug concentration. The standard error of the mean is indicated by the brackets at the top of the bars.
The relation between drug concentration and changes in food responses is not simple. This may be partly due to the fact that a "concentration-response curve" is not simply a dose-response curve, but also reflects palatability of the solution.

Schedule-induced polydipsia possesses a number of advantages not found in other procedures for inducing oral drug consump. tion. Low nonaversive dng concentrations may be used, since large liquid volumes are consumed. No liquid deprivation is necessary. Changes in the food baseline can be seen indicating the magnitude and time course of the effect of the self-administered drug. Finally, the liquid consumption is optional in relation to the food reinforced baseline. Removing the liquid does not disrupt the food responding or decrease the number of food reinforcements obtained (Meisch \& Pickens, 1968).

\section{REFERENCES}

CROSSLAND, J., \& LEONARD, B. E. Barbiturate withdrawal convulsions in the rat. Biochemical Pharmacology, supplement, 1963, 12,103.

ESSIG, C. F. Barbiturate withdrawal in white rats. International Journal of Neuropharmacology, 1966, 5, 103-107.

FALK, J. L. Production of polydipsia in normal rats by an intermittent food schedule. Science, $1961,133,195-196$.

LESTER, D. Self-maintenance of intoxication in the rat. Quarterly Joumal of Studies on Alcohol, 1961, 22, 223-231.

MEISCH, R., \& PICKENS, R. Oral self-stimulation of ethanol by the rat. Paper presented at the Psychonomic Society Meeting, St. Louis, Missouri, November, 1968.

SENTER, R. J., \& SINCLAIR, J. D. Selfmaintenance of intoxication in the rat: $A$ modified replication. Psychonomic Science.
$1967,9,291-292$.

NOTES

1. Preparation of this article was supported in part by USPHS Research Grants MH-15349, $\mathrm{MH}-8565$, and $\mathrm{MH}-14112$.

2. Life Insurance Medical Research Fellow.

\section{The effect of deprivation upon fixed ratio responding'}

ROBERT W. POWELL, University of
South Florida, Tampa, Fla. 33620

The effect of food deprivation upon fixed-ratio responding was studied in two pigeons. Post reinforcement pausing and response rate were considered separately. Both birds showed significant decreases in pausing as deprivation increased, but response rate did not show a similarly consistent correlation.

In studies of fixed-ratio (FR) behavior, it has become common practice to consider separately the post reinforcement pause and the response rate (Ferster \& Skinner, 1957; Lyon \& Felton, 1966; Powell, 1968). These and other reports have indicated that the post reinforcement pause is susceptible to influence by a number of variables, while the response rate is unaffected by the same variables.

In one of the earliest studies of FR behavior, Sidman \& Stebbins (1954) observed the effects of prefeeding and overfeeding during the session. Their results suggested that the post reinforcement pause 